\title{
Role of HLA-B Alleles and Clinical Presentation of B27 Negative Spondyloarthritis Patients from Mumbai, Western India
}

\author{
Devaraj J. Parasannanavar, ${ }^{1}$ Anjali Rajadhyaksha, ${ }^{2}$ and Kanjaksha Ghosh ${ }^{3}$ \\ ${ }^{1}$ Microbiology and Immunology Division, National Institute of Nutrition (ICMR), Jamai-Osmania, Tarnaka, Hyderabad, \\ Andhra Pradesh 500007, India \\ ${ }^{2}$ Department of Medicine, King Edward Memorial Hospital, Parel, Mumbai 400 012, India \\ ${ }^{3}$ National Institute of Immunohaematology (ICMR), King Edward Memorial Hospital, 13th Floor, N.M.S. Building, \\ Parel, Mumbai 400 012, India \\ Correspondence should be addressed to Kanjaksha Ghosh; kanjakshaghosh@hotmail.com
}

Received 14 October 2013; Accepted 30 January 2014; Published 6 March 2014

Academic Editor: Edmond J. Yunis

Copyright (C) 2014 Devaraj J. Parasannanavar et al. This is an open access article distributed under the Creative Commons Attribution License, which permits unrestricted use, distribution, and reproduction in any medium, provided the original work is properly cited.

\begin{abstract}
Seronegative spondyloarthritis (SpA) are variably associated with HLA-B ${ }^{*} 27$ antigen. HLA-B* 27 negative SpA has also been reported from different parts of the world. There is paucity of data on this entity from Indian subcontinent. We studied 100 consecutively diagnosed HLA-B27 negative spondyloarthritis patients from a tertiary care center in India. Modified New York Criteria for ankylosing spondylitis (AS) and ESSG criteria for SpA were used for diagnosing patients. HLA-B*27 typing was done by an in-house PCR-SSP technique in SpA patients to exclude B ${ }^{*} 27$ positive patients and PCR-SSOP technique was used to type 100 $\mathrm{B}^{*} 27$ negative SpA patients and 100 controls from the same ethnicity. Frequency of $\mathrm{B}^{*} 07$ was significantly increased $\left(\mathrm{B}^{*} 07: \%\right.$ PF 54 versus 18 ; OR 5.348; 95\% CI 2.808-10.186; $P$ value $1.14 E-07)$, whereas frequency of $B^{*} 40$ was significantly decreased $\left(B^{*} 40: \%\right.$ PF 17 versus 32 ; OR $0.435 ; 95 \%$ CI $0.222-0.850 ; P$ value 0.013 ) when compared with $B^{*} 27$ negative controls. Among $100 \mathrm{SpA}$ patients, 47 were undifferentiated spondyloarthritis and 33 patients were reactive arthritis patients. $40 \%$ of the patients were suffering from polyarticular arthritis, $35 \%$ had pauciarticular arthritis with knee joint, hip joint, ankle joint, and SI joint involvement. We conclude that $\mathrm{B}^{*} 07$ was significantly associated with B27 negative spondyloarthropathy from Western India and majority of $\mathrm{B}^{*} 27$ negative patients were uSpA.
\end{abstract}

\section{Introduction}

The spondyloarthritis (SpA) is a group of diseases which include mainly ankylosing spondylitis (AS), Reiter's syndrome/reactive arthritis (RS/ReA), enteropathic spondylitis (Crohn's disease and ulcerative colitis), Psoriatic Arthropathy (PsA), and undifferentiated spondylitis (uSpA). AS is a chronic inflammatory disease that begins primarily in the sacroiliac joints and goes on to involve the spine and other large joints. Reactive arthritis (ReA) is an acute nonporulant arthritis complicating an infection elsewhere in the body, usually genitourinary infection with Chlamydia trachomatis and enteritis due to gram negative enterobacteria such as Shigella, Salmonella, Yersinia, or Campylobacter species [1]. Psoriatic arthritis (PsA) refers to an inflammatory arthritis that typically occurs in individuals with Psoriasis [2]. Many patients, usually young adults, present with some features of one or more of the spondyloarthritis but lack criteria for these diagnosis; for example, a patient may present with inflammatory synovitis of one knee, achilles tendonitis, and dactylitis of one digit or sacroiliitis in the absence of other criteria for AS. Such patients are said to have undifferentiated spondyloarthritis (uSpA).

An association between HLA-B27 and AS was first reported in 1973 [3] and this was confirmed later [4-8]. The frequency of HLA-B27 ranges from 88 to $90 \%$ among patients as compared to $4-8 \%$ in controls. However, different races show different rates of association. The frequency of HLA-B27 with AS or other related SpA among Indian population varies from 30 to $94 \%$ as compared to $1.4-8 \%$ of 
TABLE 1: Distribution of HLA-B alleles among B27 negative SpA patients and controls from Western India (patients $(n=100)$; controls $(n=100))$.

\begin{tabular}{|c|c|c|c|c|c|c|c|}
\hline Serial number & HLA-B & $\% \mathrm{PF}$ & \%P.F & OR & $95 \% \mathrm{CI}$ & $\chi^{2} Y$ & $P$ value \\
\hline 1 & $\mathrm{~B}^{*} 51$ & 10 & 13 & 0.743 & $0.309-1.785$ & 0.196 & 0.506 \\
\hline 2 & $\mathrm{~B}^{*} 52$ & 7 & 8 & 0.865 & $0.301-2.485$ & 0.072 & 0.788 \\
\hline 3 & B $^{*} 07$ & 54 & 18 & 5.348 & $2.808-10.186$ & 26.584 & $1.14 E-07$ \\
\hline 4 & $\mathrm{~B}^{*} 15$ & 19 & 12 & 1.720 & $0.785-3.765$ & 1.374 & 0.171 \\
\hline 5 & $\mathrm{~B}^{*} 08$ & 12 & 14 & 0.837 & $0.366-1.914$ & 0.044 & 0.674 \\
\hline 6 & $\mathrm{~B}^{*} 18$ & 6 & 9 & 0.645 & $0.220-1.887$ & 0.288 & 0.420 \\
\hline 7 & $\mathrm{~B}^{*} 13$ & 4 & 3 & 1.347 & $0.293-6.183$ & 0.148 & 0.700 \\
\hline 8 & $B^{*} 40$ & 17 & 32 & 0.435 & $0.222-0.850$ & 5.298 & 0.013 \\
\hline 9 & $\mathrm{~B}^{*} 35$ & 33 & 44 & 0.626 & $0.353-1.113$ & 2.112 & 0.109 \\
\hline 10 & $\mathrm{~B}^{*} 57$ & 9 & 7 & 1.314 & $0.469-3.678$ & 0.067 & 0.602 \\
\hline 11 & $\mathrm{~B}^{*} 58$ & 8 & 4 & 2.080 & $0.607-7.169$ & 0.797 & 0.233 \\
\hline 12 & $\mathrm{~B}^{*} 44$ & 9 & 15 & 0.560 & $0.232-1.348$ & 1.184 & 0.191 \\
\hline 13 & $\mathrm{~B}^{*} 47$ & 3 & 6 & 0.484 & $0.117-1.995$ & 0.465 & 0.306 \\
\hline 14 & $\mathrm{~B}^{*} 48$ & 2 & 0 & 5.102 & $0.241-107.70$ & 0.505 & 0.155 \\
\hline 15 & $\mathrm{~B}^{*} 53$ & 1 & 1 & 1.000 & $0.061-16.224$ & 0.000 & 1.000 \\
\hline 16 & $B^{*} 49$ & 1 & 3 & 0.326 & $0.033-3.196$ & 0.255 & 0.312 \\
\hline 17 & $\mathrm{~B}^{*} 38$ & 0 & 1 & 0.330 & $0.013-8.205$ & 1.005 & 0.316 \\
\hline 18 & $\mathrm{~B}^{*} 78$ & 0 & 2 & 0.196 & $0.009-4.138$ & 0.505 & 0.155 \\
\hline 19 & $\mathrm{~B}^{*} 81$ & 1 & 3 & 0.326 & $0.333-3.196$ & 0.255 & 0.312 \\
\hline 20 & $\mathrm{~B}^{*} 37$ & 1 & 3 & 0.326 & $0.333-3.196$ & 0.255 & 0.312 \\
\hline 21 & $B^{*} 42$ & 0 & 1 & 0.330 & $0.013-8.205$ & 1.005 & 0.316 \\
\hline 22 & $\mathrm{~B}^{*} 56$ & 3 & 1 & 3.062 & $0.312-29.965$ & 0.255 & 0.312 \\
\hline
\end{tabular}

$\mathrm{N}+$ : number positive, PF: phenotype frequency, OR: odds ratio, $\chi^{2} \mathrm{Y}$ : chi-square with Yates' correction.

the general population [8]. However, a more number of B27 negative individuals could develop SpA with typical clinical and radiological findings. There are reports which reveal that other HLA-B locus alleles have also be involved in B27 negative SpA patients from India [9] and worldwide [1021]. Association of HLA-B7 CREG antigens (B7, B22, B27, B40, and B42) with AS patients among American Blacks was studied by Khan et al. $[10,11]$. Subsequently these findings were confirmed among ReA and uSpA patients from different parts of the world [12-15]. The association of HLA-B40 with AS was reported by Robinson et al. for the first time, who found that HLA-B60 (Split of B40) was increased in B27 positive AS patients [16]. Later these findings were confirmed by other investigators among B27 positive as well as negative SpA patients [14, 16-18]. Earlier studies also showed the association between HLA-B16 (currently B38 and B39) and HLA-B35CREG (B18, B35, B51, and B62) with HLA-B27 negative Caucasian, Japanese, and German AS patients [1921]. Hence, our aim was to study the role of HLA-B alleles and their clinical presentation in $\mathrm{B}^{*} 27$ negative $\mathrm{SpA}$ patients from Western India.

\section{Materials and Methods}

2.1. Selection Criteria for Patients and Controls. In the present study, a total of 100 consecutive $B^{*} 27$ negative SpA patients were selected according to the revised New York criteria for AS [22] and ESSG for SpA [23]. Patients were evaluated by rheumatologist at K.E.M. and various hospitals from Mumbai and reevaluated by one of us (K.G). As per the selection criteria, SpA patients fulfilled at least any 4 of the following criteria: (1) insidious onset, (2) duration $>3$ months, (3) radiological bilateral or unilateral sacroiliitis, (4) limitation of motion of the lumbar spine and chest expansion, and (5) improvement of morning stiffness with exercise and not relieved by rest. All patients were negative for $\mathrm{B}^{*} 27$ typed by in-house PCR-SSP to exclude $\mathrm{B}^{*} 27$ positivity [8]. These patients had undergone various tests including radiology of affected joints, sacroiliac joints, lumbar spine, cervical spine, full blood count with ESR, rheumatoid factor, anticitrullinated antibody (anti-CCP), and other autoimmune parameters like ANA and ds DNA. The study was carried out over a period of three years from April 2009 to March 2011. The study was approved by Ethics Committees of the institute. 100 age- and sex-matched healthy individuals which were negative for $\mathrm{B}^{*} 27$ belonging to the same socioeconomic status and ethnic background during the same period comprised the controls for this study.

\subsection{HLA Typing}

2.2.1. Molecular Typing. Genomic DNA extracted standard phenol-chloroform-isoamyl alcohol method from $5 \mathrm{~mL}$ of EDTA blood. B27 negative patients and controls were typed for B locus by polymerase chain reaction reverse line strip 
sequence-specific oligonucleotide hybridization (PCR-RLSSSOP) strips (Dynal-Reli-SSO typing). Each strip for HLAB typing carried a total of 62 immobilized SSOs. Genomic DNA was amplified using HLA-B locus specific biotinylated primers and hybridized with the SSO strips. Streptavidinhorseradish peroxidase (SA-HRP) conjugates for positive color development using hydrogen peroxide $\left(\mathrm{H}_{2} \mathrm{O}_{2}\right)$ and tetramethylbenzidine (TMB) as substrate. The alleles were determined using the pattern interpretation software (PMP) supplied with the kit [24].

2.3. Statistical Analysis. The allele frequencies, odds ratio, 95\% confidence interval, and chi-square with Yates' correction were estimated using SPSS software.

\section{Results}

Rheumatoid factor, anti-CCP antibody, ANA, and ds DNA were negative for all patients. Distribution of HLA-B alleles among $100 \mathrm{~B}^{*} 27$ negative SpA patients and 100 controls is as shown in Table 1 . The frequency of HLA- ${ }^{*} 07$ was significantly increased among patients as compared to the controls ( $B^{*} 07$ : \%PF 54 versus 18; OR 5.348; 95\% CI 2.80810.186; $\chi^{2}$ Y 26.584; $P$ value 1.14E - 07), whereas frequency of $\mathrm{B}^{*} 40$ was significantly decreased ( $\mathrm{B}^{*} 40$ : $\% \mathrm{PF} 17$ versus 32 ; OR 0.435; 95\% CI 0.222-0.850; $\chi^{2}$ Y 5.298; $P$ value 0.013 ). Clinical presentation of $\mathrm{B}^{*} 27$ negative SpA patients is as shown in Table 2; when $52 \%$ of patients who are analyzed had the onset of clinical symptom between the age of 16 to 30 years, 30\% showed the onset between the age between 31 to 45 years of age and male to female ratio of $4: 1$. Subgroup classification among $\mathrm{B}^{*} 27$ negative SpA patients revealed that $47 \%$ patients had undifferentiated spondyloarthritis, $33 \%$ were $\operatorname{ReA}, 14 \%$ were AS, and JSpA were $4 \%$. Analysis of the type of arthritis showed that $40 \%$ of the patients were suffering from polyarticular arthritis, $35 \%$ had pauciarticular arthritis, and $25 \%$ had monoarticular. Further analysis of the involvent of joints showed that $54 \%$ had knee joints affected, $46 \%$ had hip joint pain, $30 \%$ had ankle joints, and $25 \%$ of patients had SI joints involvement. When Scober's test results were analyzed, $50 \%$ of the patients had restricted spine flexion and $25 \%$ had diminished chest expansion. Enthesitis was present in $84 \%$ of the patients.

\section{Discussion}

The association between HLA-B27 with AS and related arthropathies has been known for a long time. But it is thought that in addition to B27, other HLA-B and DR alleles may increase susceptibility to the development of AS and related arthropathies [16]. The present study revealed the role of other HLA-B locus alleles and their clinical presentation among B27 negative SpA patients from Western India. In this study, HLA-B* 07 was significantly increased among B27 negative SpA patients from Western India, which confirms earlier reports [9-15]. B* 07 may be associated with AS either directly or because of linkage disequilibrium with further
TABLE 2: Clinical presentation of B27 negative SpA patients.

\begin{tabular}{|c|c|c|c|}
\hline $\begin{array}{l}\text { Serial } \\
\text { number }\end{array}$ & Character & $\begin{array}{c}\text { B27 } \\
\text { negative } \\
n=100\end{array}$ & $\begin{array}{l}\text { Normal controls } \\
\quad n=100\end{array}$ \\
\hline \multirow{5}{*}{1} & Age of onset & $\%$ & $\%$ \\
\hline & $\leq 15$ & 11 & 14 \\
\hline & $16-30$ & 52 & 21 \\
\hline & $31-45$ & 30 & 58 \\
\hline & $>45$ & 7 & 7 \\
\hline \multirow{3}{*}{2} & Sex: & & \\
\hline & $\mathrm{M}$ & 80 & 56 \\
\hline & $\mathrm{F}$ & 20 & 44 \\
\hline 3 & Fam H/O arthritis & 13 & 0 \\
\hline 4 & Polyarticular arthritis $>3$ Jts & 40 & 0 \\
\hline 5 & Pauciarticular & 35 & 0 \\
\hline 6 & Monoarticular & 25 & 0 \\
\hline 7 & Chest exp $\leq 5 \mathrm{~cm}$ & 25 & 0 \\
\hline 8 & Spine $\mathrm{flx} \leq 5 \mathrm{~cm}$ & 50 & 0 \\
\hline 9 & Tend SI JT & 25 & 0 \\
\hline 10 & Knee jt & 54 & 0 \\
\hline 11 & Ankle jt & 30 & 0 \\
\hline 12 & L-spine & 38 & 0 \\
\hline 13 & C-spine & 43 & 0 \\
\hline 14 & Hip jt & 46 & 0 \\
\hline 15 & SI jt & 25 & 0 \\
\hline 16 & Shoulder jt & 7 & 0 \\
\hline 17 & Wrist jt & 11 & 0 \\
\hline 18 & Foot jt & 7 & 0 \\
\hline 19 & Hand jt & 9 & 0 \\
\hline 20 & Enthesitis & 84 & 0 \\
\hline
\end{tabular}

MHC gene. Our findings suggest that in addition to HLA$B^{*} 27, B^{*} 07$ may also play a significant role in the development of SpA in Western Indian population.

Several studies have suggested that association of HLA$\mathrm{B}^{*} 40$ with $\mathrm{B}^{*} 27$ positive as well as negative AS patients from various populations [16-18]. However, in our study $B^{*} 40$ was decreased among patients when compared with controls. An earlier study reported that even though $\mathrm{B}^{*} 40$ frequency was increased marginally, no association was found between $B^{*} 40$ and SpA from north Indian population [24]. As HLA-B ${ }^{*} 27$ and $B^{*} 07$ are more strongly associated with the disease, hence in our cohort of SpA patients where HLA-B ${ }^{*} 27$ and $B^{*} 07$ are present in significant numbers, impact of $\mathrm{B}^{*} 40$ has become statistically less. However, there are quite a few studies where $\mathrm{B}^{*} 40$ was found to have stronger association with rheumatoid arthritis than with SpA [25], and these patients have higher tendency to have an associated pulmonary involvement. In our cohort such patients who were rheumatoid factor positive or were diagnosed with rheumatoid arthritis were meticulously excluded providing another reason for lower $B^{*} 40$ prevalence in our series. 
Spondyloarthritis patients presented, either singly or in combination, with sacroiliitis with spondylitis; polyarticular arthritis; persistent pain and tenderness in the tendo-Achillis or heel; or pauciarticular arthritis associated with recent history (three months previously) of dysentery, together with high (ESR $>40 \mathrm{~mm} / \mathrm{h}$ ) and negative autoimmune parameters (ANA, dsDNA, and ANCA). The disease lasted for more than three months and responded to nonsteroidal antiinflammatory drugs (NSAIDS) even before the diagnosis. Some patients showed systemic symptoms such as weight loss and low grade fever, 5 patients had uveitis, 4 patients had urethritis, and 2 patients had psoriatic arthropathies. Diseases at different duration revealed that majority of the patients were between age 16 to 30 years group in all durations. Chest expansion of $\leq 5 \mathrm{~cm}$ was present alone or together in $64 \%$ of the patients studied. Hence even in HLA$\mathrm{B}^{*} 27$ negative SpA patients, and this test is clinically useful. Undifferentiated form of SpA (47\%), enthesitis (84\%), and polyarticular arthritis was significantly over represented than in $\mathrm{B}^{*} 27$ associated $\mathrm{SpA}$ as reported in the literature [15]. Hence HLA-B* 27 negative SpA resembles classical HLA$\mathrm{B}^{*} 27$ positive variety of $\mathrm{SpA}$ in showing male preponderance and seronegativity (for both rheumatoid factor and antiCCP antibodies). However, it differs substantially in clinical presentation by older age of presentation, high frequency of enthesitis, and polyarticular arthritis, but much lower frequency of sacroiliitis which corroborates earlier study on American pediatric SpA patients suggests that clinician needs look closely for enthesitis to diagnose SpA [26]. In our study HLA-B* 40 was significantly underrepresented in our patients when compared to other reported studies elsewhere in the world [14-18] which suggests that environmental precipitating factors are likely to be varied in different parts of the world, explaining the differential presentation of HLA$\mathrm{B}^{*} 07$ and HLA-B ${ }^{*} 40$ in these groups of patients.

\section{Conflict of Interests}

The authors declare that there is no conflict of interests regarding the publication of this paper.

\section{Acknowledgments}

This study was presented as poster presentation at 1st Meeting of Asian-Pacific Federation of Inflammation and Regeneration: APFIR-2011 which was held on June 2-3, 2011, at Kyoto, Japan. The authors acknowledge Professor (Dr.) N. K. Mehra, Department of Transplant Immunology and Immunogenetics, All India Institute of Medical Sciences, New Delhi, for his critical comments, valuable suggestions, and recommendations.

\section{References}

[1] R. A. Hughes and A. C. Keat, "Reiter's syndrome and reactive arthritis: a current view," Seminars in Arthritis and Rheumatism, vol. 24, no. 3, pp. 190-210, 1994.

[2] J. M. H. Moll and V. Wright, "Psoriatic arthritis," Seminars in Arthritis and Rheumatism, vol. 3, no. 1, pp. 55-78, 1973.
[3] D. A. Brewerton, F. D. Hart, A. Nicholls, M. Caffrey, D. C. James, and R. D. Sturrock, "Ankylosing spondylitis and HL-A 27," The Lancet, vol. 301, no. 7809, pp. 904-907, 1973.

[4] C. Lopez-Larrea, K. Sujirachato, N. K. Mehra et al., "HLAB27 subtypes in Asian patients with ankylosing spondylitis: evidence for new associations," Tissue Antigens, vol. 45, no. 3, pp. 169-176, 1995.

[5] S. R. Kankonkar, S. C. Raikar, S. V. Joshi, and S. J. Tijoriwala, "Association of HLA-B27 antigen in Indian patients of ankylosing spondylitis and other autoimmune diseases," Journal of Association of Physicians of India, vol. 46, no. 4, pp. 345-350, 1998.

[6] U. Shankarkumar, J. P. Devraj, K. Ghosh, and D. Mohanty, "Seronegative spondarthritis and human leucocyte antigen association," British Journal of Biomedical Science, vol. 59, no. 1, pp. 38-41, 2002.

[7] U. Shankarkumar, K. Ghosh, and D. Mohanty, "Novel HLA $\mathrm{B}^{*} 2714$ and $\mathrm{B}^{*} 2708$ allele associations in seronegative spondarthritis patients and haemophilia patients with chronic synovitis in India," Tissue Antigens, vol. 62, no. 2, pp. 175-178, 2003.

[8] J. P. Devraj, A. Rajyadhaksha, and K. Ghosh, "Application of a simple in-house PCR-SSP technique for HLA-B* 27 typing in Spondyloarthropathies (SpA) patients," Arthritis, vol. 2013, Article ID 504109, 4 pages, 2013.

[9] J. P. Devraj, U. Shankarkumar, and K. Ghosh, "Increased frequency of HLA-B7 among B27-negative seronegative spondarthritis patients from Mumbai, Western India," British Journal of Biomedical Science, vol. 66, no. 1, pp. 25-27, 2009.

[10] M. A. Khan, I. Kushner, and W. E. Braun, "A subgroup of ankylosing spondylitis associated with HLA-B7 in American blacks," Arthritis and Rheumatism, vol. 21, no. 5, pp. 528-530, 1978.

[11] M. A. Khan, "B7-CREG and ankylosing spondylitis," British Journal of Rheumatology, vol. 22, no. 4, supplement 2, pp. 129133, 1983.

[12] F. C. Arnett Jr., M. C. Hochberg, and W. B. Bias, "Crossreactive HLA antigens in B27-negative Reiter's syndrome and sacroiliitis," The Johns Hopkins Medical Journal, vol. 141, no. 4, pp. 193-197, 1977.

[13] E. Ben-Chetrit, C. Brautbar, and A. Rubinow, "HLA antigens in Reiter's syndrome in Israeli patients," The Journal of Rheumatology, vol. 12, no. 5, pp. 964-966, 1985.

[14] J.-P. Cedoz, D. Wendling, and J.-F. Viel, “The B7 cross reactive group and spondyloarthropathies: an epidemiological approach," The Journal of Rheumatology, vol. 22, no. 10, pp. 1884-1890, 1995.

[15] P. D. Sampaio-Barros, R. A. Conde, E. A. Donadi et al., "Undifferentiated spondyloarthropathies in Brazilians: importance of HLA-B27 and the B7-CREG alleles in characterization and disease progression," The Journal of Rheumatology, vol. 30, no. 12, pp. 2632-2637, 2003.

[16] W. P. Robinson, S. M. van der Linden, M. A. Khan et al., "HLABw60 increases susceptibility to ankylosing spondylitis in HLAB27+ patients," Arthritis and Rheumatism, vol. 32, no. 9, pp. 1135-1141, 1989.

[17] J. C. C. Wei, W. C. Tsai, H. S. Lin, C. Y. Tsai, and C. T. Chou, "HLA-B60 and B61 are strongly associated with ankylosing spondylitis in HLA-B27-negative Taiwan Chinese patients," Rheumatology, vol. 43, no. 7, pp. 839-842, 2004.

[18] M. A. Khan, I. Kushner, and W. E. Braun, "B27-negative HLABW16 in ankylosing spondylitis," The Lancet, vol. 311, no. 8078, pp. 1370-1371, 1978. 
[19] A. Yamaguchi, N. Tsuchiya, H. Mitsui et al., "Association of HLA-B39 with HLA-B27-negative ankylosing spondylitis and pauciarticular juvenile rheumatoid arthritis in Japanese patients: evidence for a role of the peptide-anchoring B pocket," Arthritis and Rheumatism, vol. 38, no. 11, pp. 1672-1677, 1995.

[20] P. Wagener, H. Zeidler, G. Eckert, and H. Deicher, "Increased frequency of HLA-Bw35 CREG antigens in HLA-B27 negative ankylosing spondylitis," British Journal of Rheumatology, vol. 22, no. 4, pp. 134-135, 1983.

[21] S. van der Linden, H. A. Valkenburg, and A. Cats, "Evaluation of diagnostic criteria for ankylosing spondylitis. A proposal for modification of the New York criteria," Arthritis and Rheumatism, vol. 27, no. 4, pp. 361-368, 1984.

[22] M. Dougados, S. van der Linden, R. Juhlin et al., "The European spondylarthropathy study group preliminary criteria for the classification of spondylarthropathy," Arthritis and Rheumatism, vol. 34, no. 10, pp. 1218-1227, 1991.

[23] V. Agrawal, R. M. Gupta, U. Usha, and S. V. Sharma, "HLA class-I and class-II antigen association in rheumatoid arthritis at Varanasi, India," Indian Journal of Pathology and Microbiology, vol. 39, no. 1, pp. 19-25, 1996.

[24] U. Shankarkumar, D. Prasanavar, K. Ghosh, and D. Mohanty, "HLA-A* 02 allele frequencies and B haplotype associations in Western Indians," Human Immunology, vol. 64, no. 5, pp. 562566, 2003.

[25] J. Seignalet, F. Blotman, L. Simon, and B. Soustelle, "HLA-A, $\mathrm{B}, \mathrm{C}$, and DR typing in rheumatoid arthritis," La Revue de Médecine Interne, vol. 5, no. 2, pp. 128-135, 1984.

[26] M. L. Stoll, R. Bhore, M. Dempsey-Robertson, and M. Punaro, "Spondyloarthritis in a pediatric population: risk factors for sacroiliitis," The Journal of Rheumatology, vol. 37, no. 11, pp. 2402-2408, 2010. 


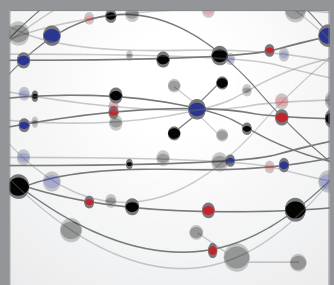

The Scientific World Journal
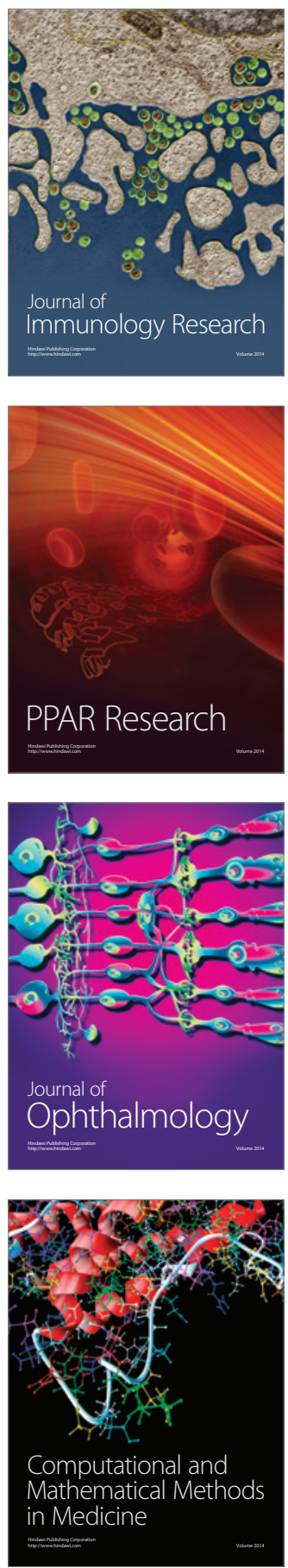

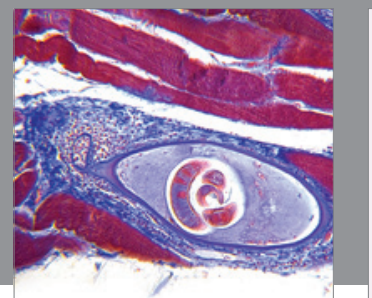

Gastroenterology

Research and Practice
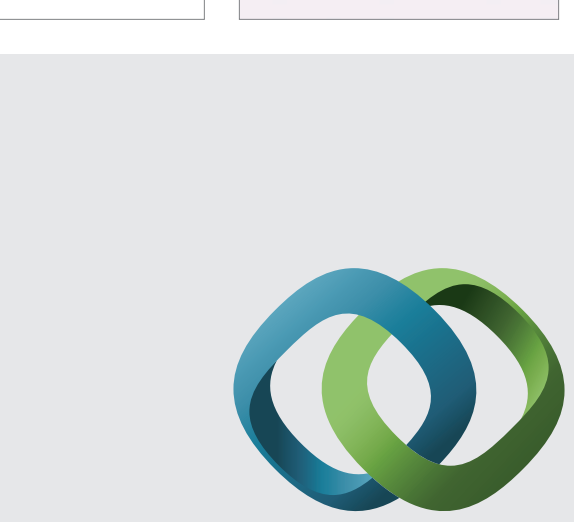

\section{Hindawi}

Submit your manuscripts at

http://www.hindawi.com
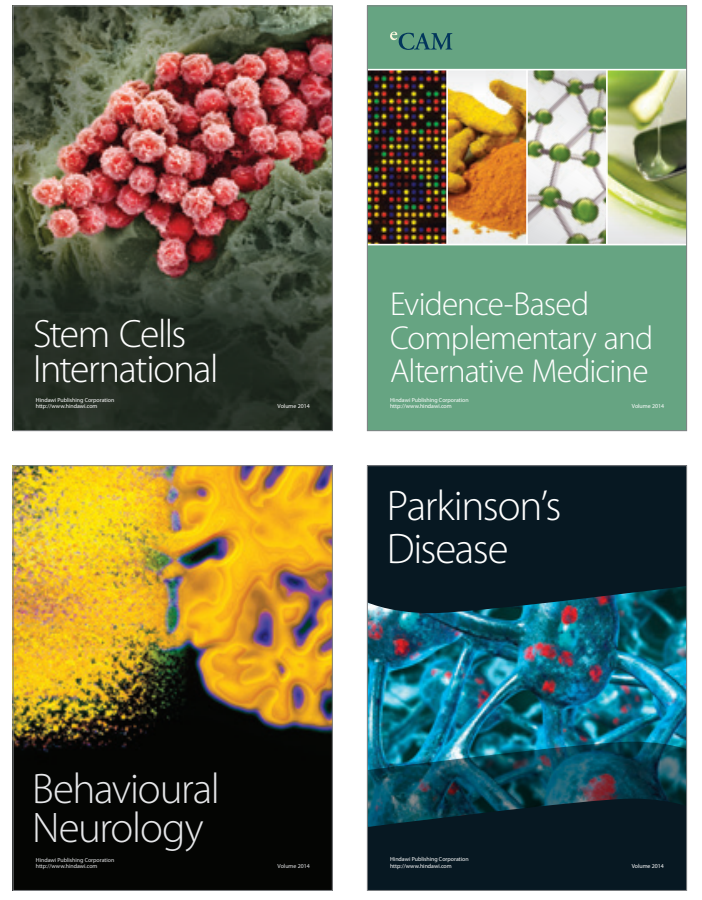
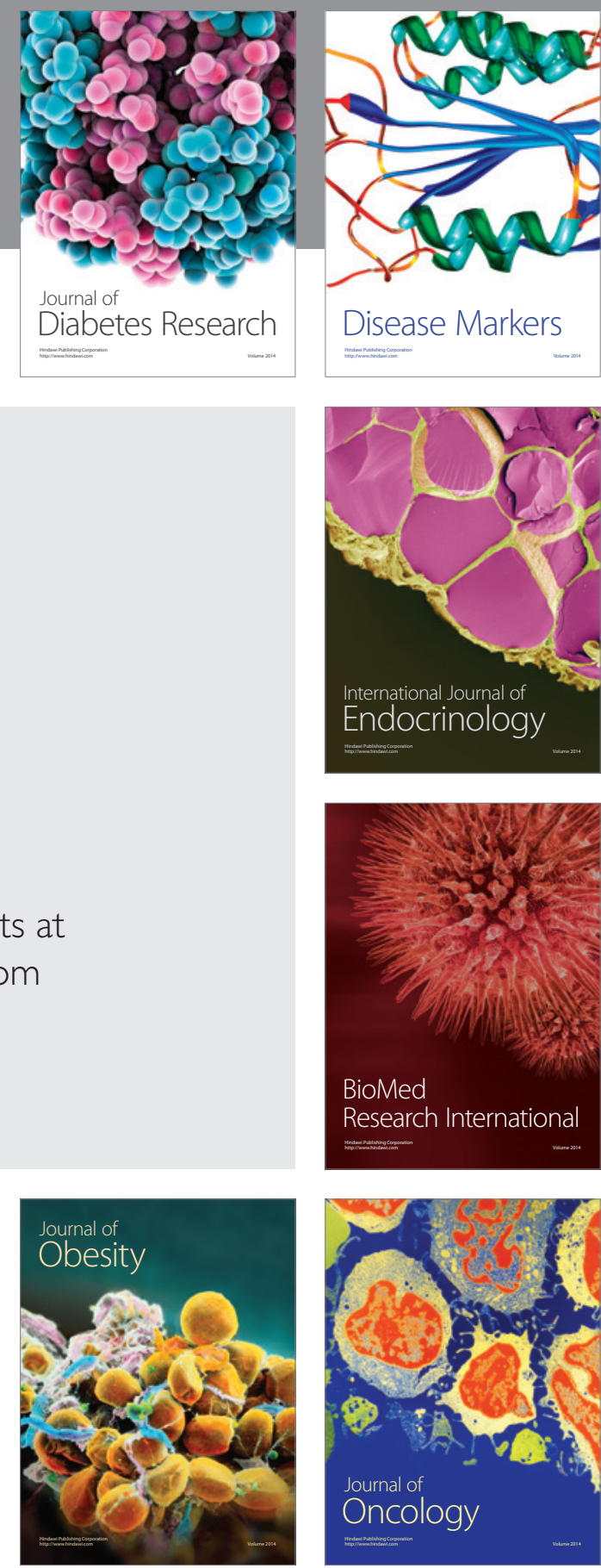

Disease Markers
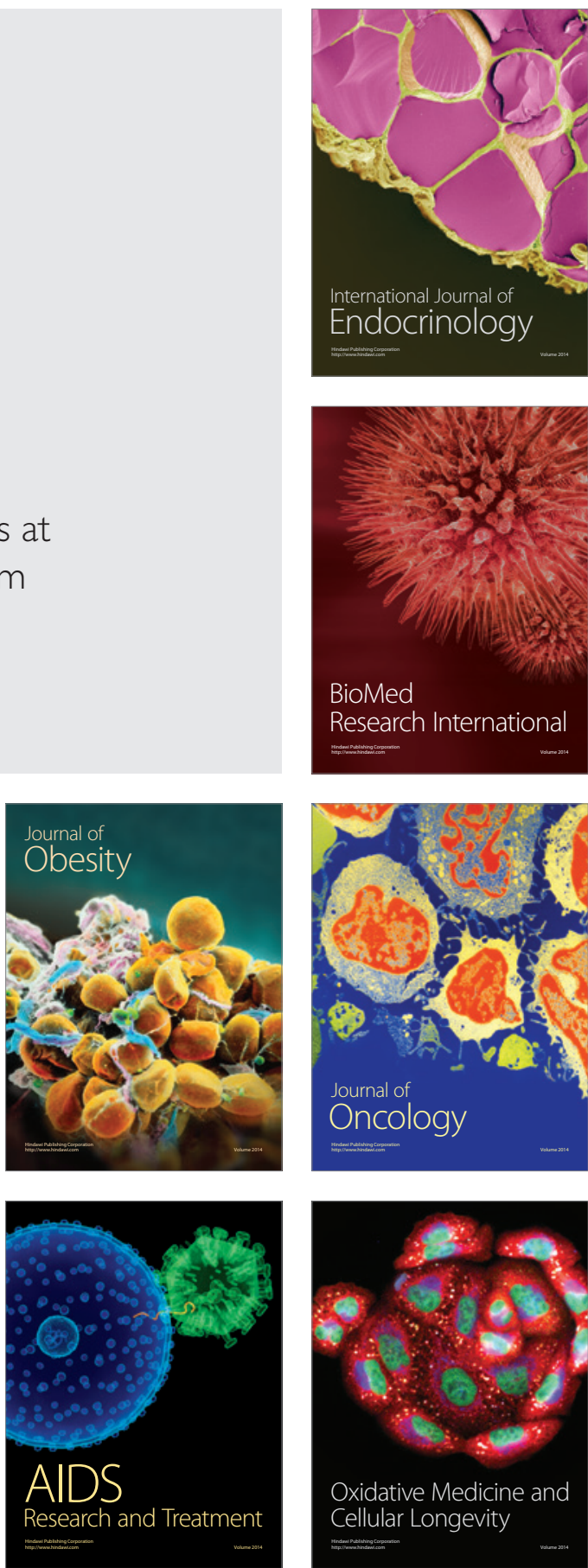